\title{
Effect of the human cumulus oophorus on movement characteristics of human capacitated spermatozoa
}

\author{
J. Tesařík, C. Mendoza Oltras* and J. Testart \\ INSERM, Unité 187, Service de Gynécologie-Obstétrique, Hôpital Antoine Béclère, 92141 Clamart, \\ France; and ${ }^{*}$ Department of Biochemistry, University of Granada, Spain
}

\begin{abstract}
Summary. The effect of human cumulus oophorus on movement characteristics of human spermatozoa previously incubated in vitro under capacitating conditions was studied using automated digital image analysis. When spermatozoa were incubated for a short time with whole cumuli, most of those that penetrated the cumulus intercellular matrix were characterized by a linear movement with small amplitudes of lateral head displacement, but with elevated values of beat cross frequency. Short $(5 \mathrm{~min})$ incubation with solubilized cumulus intercellular matrix of spermatozoa preincubated in capacitating conditions $(6 \mathrm{~h})$ significantly reduced the percentage of spermatozoa showing the 'hyperactivated' type of motility characterized by high curvilinear velocity, low progressive velocity and elevated values of lateral head displacement. Moreover, a subpopulation of spermatozoa with very high values of progressive velocity and beat cross fequency and with reduced amplitudes of lateral head displacement appeared in these conditions. This cumulus-related motility pattern was not seen in fresh spermatozoa or in those incubated in the absence of cumulus material. Changes in the sperm movement characteristics similar to those observed in the presence of the solubilized cumulus matrix could also be induced by some of its h.p.l.c. fractions. These results show that the intercellular matrix of the human cumulus oophorus exerts a specific effect on human sperm motility, probably acting preferentially on the 'hyperactivated' sperm subpopulation.
\end{abstract}

Keywords: cumulus oophorus; sperm motility; digital image analysis; capacitation; human

\section{Introduction}

It has been shown in various mammalian species that the ability of spermatozoa to change the character of movement in response to different environmental conditions is an important facet of their fertilizing ability (for review see Yanagimachi, 1988). Typical changes in sperm movement characteristics occur during sperm capacitation. When human spermatozoa are incubated in vitro in capacitating media, motility changes resembling those observed in spermatozoa of some other mammalian species can be observed (Burkman, 1984; Mortimer et al., 1984; Morales et al., 1988; Robertson et al., 1988). In general, these changes are characterized by the appearance of a distinct subpopulation of spermatozoa that display a vigorous motility but little progression with large amplitudes of lateral head displacement. It has been suggested that this so-called 'hyperactivated' motility is typical of capacitated spermatozoa and that it shortly precedes the acrosome reaction (Robertson et al., 1988). If this presumption is true, the hyperactivated motility should be acquired by the fertilizing spermatozoon close to, or during, its interaction with the egg investments - the cumulus oophorus and the zona pellucida. However, no data have been published concerning the character of movement of human spermatozoa within the human cumulus oophorus and the observations reported for other mammalian species are somewhat inconsistent (Suarez et al., 1983; Katz et al., 1986; Corselli \& Talbot, 1987; Drobnis et al., 1988; Shalgi \& Phillips, 1988). 
In this study we analysed the movement characteristics of human spermatozoa preincubated in capacitating medium and allowed to penetrate the human cumulus oophorus. To distinguish whether the observed changes in sperm motility pattern were merely due to mechanical interaction of spermatozoa with the cumulus microstructure or whether a direct specific effect of cumulus components on the character of sperm movement also contributed to them, the solubilized cumulus intercellular matrix as well as its different fractions separated by high-performance liquid chromatography (h.p.l.c.) were tested for their effects on human sperm motility.

\section{Materials and Methods}

\section{Semen collection and preparation}

Semen samples were obtained from 12 normospermic (World Health Organization, 1987) men from couples treated by in-vitro fertilization. Spermatozoa were prepared for in-vitro fertilization using the standard protocol (Testart et al., 1982). Briefly, semen was left to stand for 20-30 min to allow liquefaction. An aliquant of $0.5 \mathrm{ml}$ semen was then diluted with $9 \mathrm{ml}$ Tyrode's medium (Difco, Detroit, MI, USA) and spermatozoa were washed from the seminal plasma by two cycles of centrifugation and resuspension of the sperm pellet in the medium. For selection of motile spermatozoa, the final suspension was centrifuged again, $300 \mu \mathrm{l}$ of B2 medium (API System, MontalieuVercieu, France) were carefully layered onto the pellet and the tubes were left for $30 \mathrm{~min}$ at $37^{\circ} \mathrm{C}$ in an incubator filled with $5 \% \mathrm{CO}_{2}$ in air. The supernatant containing the most active spermatozoa was then decanted. Only spermatozoa from samples allowing fertilization and cleavage of at least one oocyte were used. For in-vitro capacitation washed spermatozoa were incubated for $2-6 \mathrm{~h}$ in $\mathrm{B} 2$ medium. This incubation was carried out at $37^{\circ} \mathrm{C}$ and under the gas phase of $5 \% \mathrm{CO}_{2}$ in air.

\section{Source and preparation of cumuli oophori}

Source of cumuli. Transvaginal ultrasonically guided follicular aspiration was performed as part of the clinical in-vitro fertilization procedures. Ovarian stimulation was induced by gonadotrophins after administration of a GnRH agonist (Testart et al., 1989). Parts of the cumulus oophorus were isolated from follicular aspirates. Only fully mucified cumuli, rated as preovulatory (Laufer et al., 1984), were used. The oocytes from the respective follicles were rated as grade 3 of oocyte maturation (Marrs et al., 1984). The cumuli were kept in B2 medium at $37^{\circ} \mathrm{C}$ and under $5 \%$ $\mathrm{CO}_{2}$ in air for a period ranging between $30 \mathrm{~min}$ and $2 \mathrm{~h}$. They were then washed thoroughly by passing each cumulus fragment through three baths of Dulbecco's phosphate-buffered saline (DPBS; Gibco, Paisley, UK). The volume of each bath was $2 \mathrm{ml}$ and the time of incubation was $5 \mathrm{~min}$. Cumuli from 41 follicles punctured in 36 patients were used. About one quarter of this cumulus material was used as a whole whereas the other cumuli were processed for isolation of the intercellular matrix.

Preparation of solubilized cumulus intercellular matrix. Cumuli were dispersed by incubation for 15 min with $0.5 \mathrm{mg}$ testicular hyaluronidase (Sigma, La Verpillière, France) diluted in $1 \mathrm{ml}$ DPBS. Cumulus cells were sedimented by mild centrifugation ( $200 \mathrm{~g} ; 10 \mathrm{~min}$ ) and the supernatant containing the solubilized cumulus intercellular matrix was decanted, freeze-dried, and stored for $1-6$ weeks at $-40^{\circ} \mathrm{C}$. The contribution of hyaluronidase to the protein concentration of this preparation was less than $1 \%$ as determined by h.p.l.c. Viability assessment was performed for the sedimented cumulus cells using supravital staining with $0.04 \%$ Trypan blue in DPBS. No differences in the proportion of viable cells were found between free cumulus fragments found in follicular aspirates and parts of cumuli dissected from oocyte cumulus complexes from the same aspirates.

Chromatography. Aliquants of the freeze-dried cumulus matrix originating from different follicles were dissolved in DPBS, pooled and separated by h.p.l.c. using a Bio-Sil TSK-400 (Bio-Rad, München, FRG) gel filtration column and DPBS as eluent. The flow rate was $1 \mathrm{ml} / \mathrm{min}$. The h.p.l.c. system was calibrated with blue dextran (Sigma) for void volume and with a Bio-Rad gel filtration testmix containing the following proteins of known molecular weights: thyroglobulin (bovine), 670000; immunoglobulin G (bovine), 158000 ; ovalbumin (chicken), 44 000; myoglobin (horse), 17 000; and cyanocobalamin, 1350. Ten protein fractions were collected.

\section{Sperm incubations with cumulus materials}

Sperm incubation with parts of the whole cumulus oophorus. Individual cumuli were placed in $1 \mathrm{ml}$ of B2 medium containing $1 \times 10^{6}$ spermatozoa previously incubated for $6 \mathrm{~h}$ in this medium and incubated for an additional $5 \mathrm{~min}$ at $37^{\circ} \mathrm{C}$ under $5 \% \mathrm{CO}_{2}$ in air. This time was sufficient for spermatozoa to penetrate the cumuli, but the physical integrity of the cumulus fragments was still retained (longer incubations led to gradual disintegration of cumuli due to solubilization of the intercellular matrix). Cumuli were then briefly washed in DPBS, placed in the Makler chamber (depth $10 \mu \mathrm{m}$ ) and introduced into the motility analyser (see below) for immediate measurement. In parallel, samples of the 
same preincubation sperm populations (not added to the cumulus) were evaluated and served as controls in this experiment.

Sperm incubation with solubilized cumulus matrix. Aliquants $(50 \mu \mathrm{l})$ of sperm suspensions (sperm concentration between 1 and $5 \times 10^{6} / \mathrm{ml}$ ) were mixed with the same volume of the solubilized cumulus intercellular matrix or of its individual h.p.l.c. fractions. These materials had been previously diluted in DPBS to a final concentration of $\sim 0.5 \mathrm{mg} / \mathrm{ml}$. Incubations were carried out at $37^{\circ} \mathrm{C}$ for $5 \mathrm{~min}$. Cumulus preparations were replaced with $10 \mu \mathrm{g}$ hyaluronidase/ $\mathrm{ml}$ in control incubations.

\section{Sperm motility analysis}

All analyses were performed using the HTM motility analyzer (Hamilton Thorn Research, Inc., Danvers, MA, USA). The conditions of measurement and the setup of the main analysis parameters are summarized in Table 1. Tracks of at least 200 spermatozoa were recorded for each sample involving the solubilized cumulus matrix. Tracks of at least 30 spermatozoa not colliding with cumulus cells were recorded for each sample involving the whole cumulus oophorus. The following characteristics of sperm movement were determined for each track: curvilinear velocity (VCL), progressive velocity $(\mathrm{VSL})$, linearity $(\mathrm{LIN}=\mathrm{VSL} / \mathrm{VCL})$, beat cross frequency $(\mathrm{BCF})$, and average lateral head displacement (ALH) (for explanation of terms see Mortimer et al., 1988). At least 8 sperm samples from different donors were analysed in each experimental group. Raw numerical data characterizing tracks of individual spermatozoa were transported to a personal computer and processed using the StatView ${ }^{\circledR}$ II package (Abacus Concepts, Inc., Berkeley, CA, USA). These additional operations involved application of sort functions on different parameters of sperm motility, which made it possible to select specific sperm subpopulations and determine their quantitative representation (see 'Results'), and statistical analysis.

Table 1. Conditions of measurement and parameter set-up of the HTM image analysis system

\begin{tabular}{ll}
\hline Temperature $\left({ }^{\circ} \mathrm{C}\right)$ & $37 \cdot 5$ \\
Diluent:sample & $0: 1$ \\
Chamber & Makler $(10 \mu \mathrm{m})$ \\
Image type & Phase contrast \\
Field selection & Select \\
Calculate ALH & Yes \\
Beat frequency & Yes \\
Automatic sort & No* \\
Frames at frame rate & 20 at $25 / \mathrm{sec}$ \\
$\begin{array}{l}\text { Minimum contrast (arbitrary } \\
\text { units) }\end{array}$ & 8 \\
$\begin{array}{l}\text { Minimum size (pixels) } \\
\text { LO/HI size gates (pixels) }\end{array}$ & 6 \\
$\begin{array}{l}\text { LO/HI intensity gates (arbitrary } \\
\text { units) }\end{array}$ & $0 \cdot 5 / 2 \cdot 0$ \\
Minimum number of data points & $0 \cdot 5 / 2 \cdot 0$ \\
acquired in the track & 14 \\
*Sort functions were performed on raw output data \\
using the StatView II computing program.
\end{tabular}

\section{Statistical analysis}

Data were analysed by Student's unpaired $t$ test and $\chi^{2}$ test for normally distributed populations. Pearson and likelihood ratio $\chi^{2}$ analysis were used to determine significance between non-normally distributed groups.

\section{Results}

\section{Movement characteristics of spermatozoa within the intact cumulus oophorus}

When spermatozoa was preincubated for $6 \mathrm{~h}$ in capacitating medium and then allowed to penetrate the cumulus intercellular matrix, marked changes in the mean values of all the motility 
parameters measured were observed (Table 2). In general, the spermatozoa within the cumulus moved less rapidly than those in medium, but their movement was more linear with low amplitudes of lateral head displacement and with elevated values of beat cross frequency. This type of motility (Fig. la) was seen in about $90 \%$ of spermatozoa within the cumuli oophori. About $10 \%$ spermatozoa within the cumuli showed little progessive movement with higher lateral excursions of the head and low values of progressive velocity and linearity (Fig. Ib).

Table 2. Movement characteristics of spermatozoa preincubated in capacitating medium $(6 \mathrm{~h})$ and having penetrated the cumulus oophorus $(\mathrm{CO})$ as compared with control spermatozoa of the same preincubated populations (not added to the cumulus)

\begin{tabular}{lccccc}
\hline Spermatozoa & $\begin{array}{c}\text { VCL } \\
(\mu \mathrm{m} / \mathrm{sec})\end{array}$ & $\begin{array}{c}\text { VSL } \\
(\mu \mathrm{m} / \mathrm{sec})\end{array}$ & $\begin{array}{c}\text { LIN } \\
(\%)\end{array}$ & $\begin{array}{c}\text { BCF } \\
(\mathrm{Hz})\end{array}$ & $\begin{array}{c}\text { ALH } \\
(\mu \mathrm{m})\end{array}$ \\
\hline Within CO* & $61 \cdot 0 \pm 21 \cdot 0^{\mathrm{b}}$ & $48 \cdot 4 \pm 17 \cdot 9^{\mathrm{b}}$ & $78 \cdot 6 \pm 18 \cdot 8^{\mathrm{a}}$ & $17 \cdot 8 \pm 2 \cdot 9^{\mathrm{b}}$ & $3.6 \pm 1 \cdot 3^{\mathrm{b}}$ \\
Control & $101 \cdot 9 \pm 29 \cdot 7$ & $70 \cdot 1 \pm 22 \cdot 2$ & $70 \cdot 0 \pm 18 \cdot 2$ & $15 \cdot 2 \pm 2 \cdot 7$ & $6 \cdot 1 \pm 2 \cdot 2$ \\
\hline
\end{tabular}

Values given are means \pm s.d.

*Significance of differences as compared with control: ${ }^{a} P<0.01 ;{ }^{b} P<0.001$.

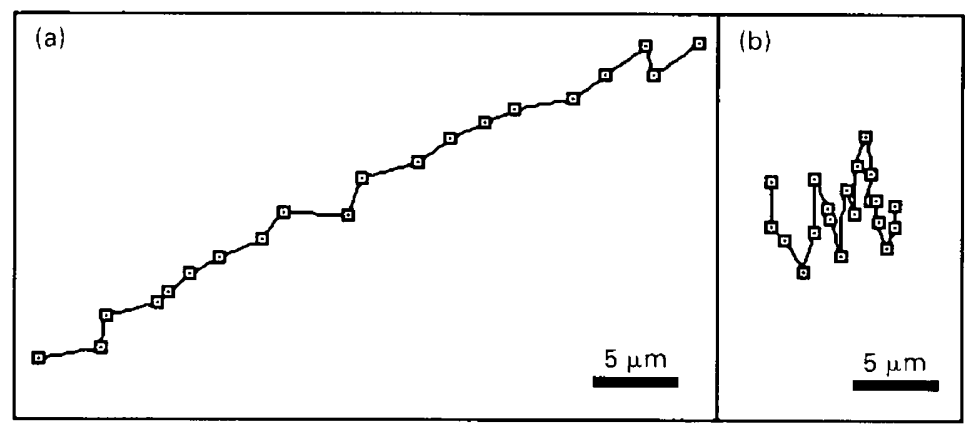

Fig. 1. Typical tracks of human spermatozoa within the cumulus oophorus. Points represent 20 consecutive positions of the sperm head recorded in $40-\mathrm{msec}$ intervals. The progressive movement of the spermatozoon in (a) is related to its low amplitude of lateral head displacement $(\mathrm{ALH}=3.2 \mu \mathrm{m})$. By contrast, the great lateral head excursions of the spermatozoon in (b) $(A L H=7 \cdot 3 \mu \mathrm{m})$ apparently restrain its motility in the viscous cumulus intercellular matrix.

\section{Effects of solubilized cumulus intercellular matrix on sperm movement characteristics}

The changes in mean values of sperm movement characteristics occurring during sperm incubation in capacitating medium and during the following incubation with the solubilized cumulus intercellular matrix are summarized in Table 3. Compared with briefly incubated samples $(30 \mathrm{~min}$ for swim-up motility selection only), longer incubation of spermatozoa in capacitating medium led to a significant increase in the average values of curvilinear velocity and lateral head displacement and to a decrease in linearity, whereas the values of progressive velocity and beat cross frequency remained unchanged. The subsequent incubation with the solubilized cumulus intercellular matrix reduced the mean amplitude of lateral head displacement and augmented the average values of progressive velocity, linearity and beat cross frequency as compared with preincubated spermatozoa subsequently exposed to hyaluronidase alone, the enzyme used for solubilization of the cumulus matrix. These changes were not observed when washed and motility-selected spermatozoa were exposed to the solubilized cumulus matrix without previous incubation for in-vitro capacitation (Table 3). The changes in sperm movement characteristics induced by the solubilized 
cumulus matrix in capacitated sperm populations were even more expressed when only highly motile subpopulations (VCL $\geq 100 \mu \mathrm{m} / \mathrm{sec}$ ) of spermatozoa were included in these calculations (Table 4). No differences in the percentage of motile spermatozoa were observed between sperm populations after the same time of incubation.

Table 3. Effects of sperm incubation in capacitating medium (B2) and their subsequent exposure to solubilized cumulus oophorus intercellular matrix (SCM) on sperm movement characteristics

\begin{tabular}{lccccc}
\hline Sperm incubation* & $\begin{array}{c}\text { VCL } \\
(\mu \mathrm{m} / \mathrm{sec})\end{array}$ & $\begin{array}{c}\text { VSL } \\
(\mu \mathrm{m} / \mathrm{sec})\end{array}$ & $\begin{array}{c}\text { LIN } \\
(\%)\end{array}$ & $\begin{array}{c}\text { BCF } \\
(\mathrm{Hz})\end{array}$ & $\begin{array}{c}\text { ALH } \\
(\mu \mathrm{m})\end{array}$ \\
\hline B2 $(30 \mathrm{~min})^{\mathrm{a}}+$ Hya $(5 \mathrm{~min})$ & $81 \cdot 2 \pm 15 \cdot 1$ & $66 \cdot 0 \pm 13 \cdot 6$ & $80 \cdot 4 \pm 16 \cdot 1$ & $14 \cdot 7 \pm 2 \cdot 3$ & $4 \cdot 4 \pm 1 \cdot 5$ \\
B2 $(30 \mathrm{~min})^{\mathrm{a}}+$ SCM $(5 \mathrm{~min})$ & $82 \cdot 1 \pm 15 \cdot 3$ & $66 \cdot 2 \pm 13 \cdot 5$ & $80 \cdot 0 \pm 15 \cdot 8$ & $14 \cdot 4 \pm 2 \cdot 3$ & $4 \cdot 5 \pm 1 \cdot 6$ \\
B2 (6 h) + Hya (5 min) & $99 \cdot 0 \pm 20 \cdot 2^{\mathrm{b}}$ & $69 \cdot 5 \pm 16 \cdot 7$ & $70 \cdot 9 \pm 14 \cdot 4^{\mathrm{b}}$ & $15 \cdot 1 \pm 2 \cdot 4$ & $6 \cdot 0 \pm 2 \cdot 1^{\mathrm{b}}$ \\
B2 (6 h) + SCM (5 min) & $99 \cdot 9 \pm 20 \cdot 3$ & $74 \cdot 0 \pm 16 \cdot 9^{\mathrm{c}}$ & $75 \cdot 0 \pm 13 \cdot 6^{\mathrm{c}}$ & $17 \cdot 2 \pm 2 \cdot 5^{\mathrm{c}}$ & $4 \cdot 9 \pm 1 \cdot 6^{\mathrm{c}}$ \\
\hline
\end{tabular}

Hyaluronidase (Hya) at the same concentration as in cumulus matrix preparations was added to B2 for the last 5 min of the control incubations.

Values given are means \pm s.d.

${ }^{a}$ Incubation for swim-up motility selection.

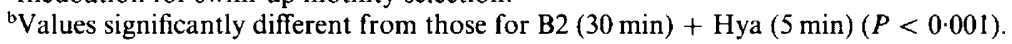

'Values significantly different from those for B2 $(6 \mathrm{~h})+$ Hya $(5 \mathrm{~min})(P<0.05$ for VSL and LIN; $P<0.001$ for BCF and ALH).

Table 4. Data from Table 3 recalculated for the sperm subpopulation with VCL $\geq 100 \mu \mathrm{m} / \mathrm{sec}$

\begin{tabular}{lccccc}
\hline Sperm incubation & $\begin{array}{c}\text { VCL } \\
(\mu \mathrm{m} / \mathrm{sec})\end{array}$ & $\begin{array}{c}\text { VSL } \\
(\mu \mathrm{m} / \mathrm{sec})\end{array}$ & $\begin{array}{c}\text { LIN } \\
(\%)\end{array}$ & $\begin{array}{c}\text { BCF } \\
(\mathrm{Hz})\end{array}$ & $\begin{array}{c}\text { ALH } \\
(\mu \mathrm{m})\end{array}$ \\
\hline B2 $(6 \mathrm{~h})+$ Hya $(5 \mathrm{~min})$ & $114 \cdot 8 \pm 10 \cdot 8$ & $75 \cdot 7 \pm 17 \cdot 6$ & $66 \cdot 7 \pm 18 \cdot 3$ & $15 \cdot 0 \pm 3 \cdot 3$ & $7 \cdot 5 \pm 2 \cdot 9$ \\
B2 $(6 \mathrm{~h})+$ SCM $(5 \mathrm{~min})$ & $113 \cdot 8 \pm 11 \cdot 8$ & $87 \cdot 7 \pm 13 \cdot 8^{\mathrm{a}}$ & $77 \cdot 8 \pm 13 \cdot 6^{\mathrm{a}}$ & $19 \cdot 8 \pm 2 \cdot 4^{\mathrm{a}}$ & $5 \cdot 2 \pm 2 \cdot 4^{\mathrm{a}}$ \\
\hline
\end{tabular}

This sort function has not been performed on data for non-incubated spermatozoa. The relative sizes of the sort fractions with VCL $\geq 100 \mu \mathrm{m} / \mathrm{sec}$ ranged between $38 \%$ and $57 \%$ of the total sperm populations and were similar in both types of incubation. Values given are means \pm s.d.

${ }^{a}$ Values significantly different from those for B2 $(6 \mathrm{~h})+$ Hya $(5 \mathrm{~min})(P<0.001)$.

In addition to the above changes in the mean values of sperm movement characteristics, the exposure of spermatozoa to the solubilized cumulus intercellular matrix brought about considerable shifts in the incidence of spermatozoa showing different values of the individual movement parameters (Fig. 2). The most outstanding features of the cumulus-exposed sperm populations were an increase in the incidence of spermatozoa showing VSL $>80 \mu \mathrm{m} / \mathrm{sec}(P<0.01)$ (Fig. 2a) and $\mathrm{BCF}>18 \mathrm{~Hz}(P<0.01)$ (Fig. 2c), and a decrease in the incidence of spermatozoa with LIN $\leqslant 60 \%(P<0.01)$ (Fig. 2b) and ALH $>7 \mu \mathrm{m}(P<0.01)$ (Fig. 2d).

These findings made it possible to distinguish two typical motility patterns, given by combination of different parameter range restrictions (see Fig. 3 legend). The one, referred to as hyperactivated pattern, was represented only sporadically at the beginning of sperm incubation in capacitating medium, but its frequency grew during the time of incubation. The incidence of spermatozoa showing the hyperactivated pattern dropped abruptly after the brief exposure of the preincubated spermatozoa to the solubilized cumulus intercellular matrix (Fig. 3). Concomitantly with the sharp reduction of the incidence of the hyperactivated motility pattern, the other pattern, for which we used the term 'cumulus-related', made its appearance in the presence of the cumulus matrix (Fig. 3). This pattern of sperm motility was never observed in fresh spermatozoa or in those incubated in capacitating medium in the absence of cumulus materials. Typical track reconstructions of non-hyperactivated, hyperactivated and cumulus-related spermatozoa are shown in Fig. 4. 

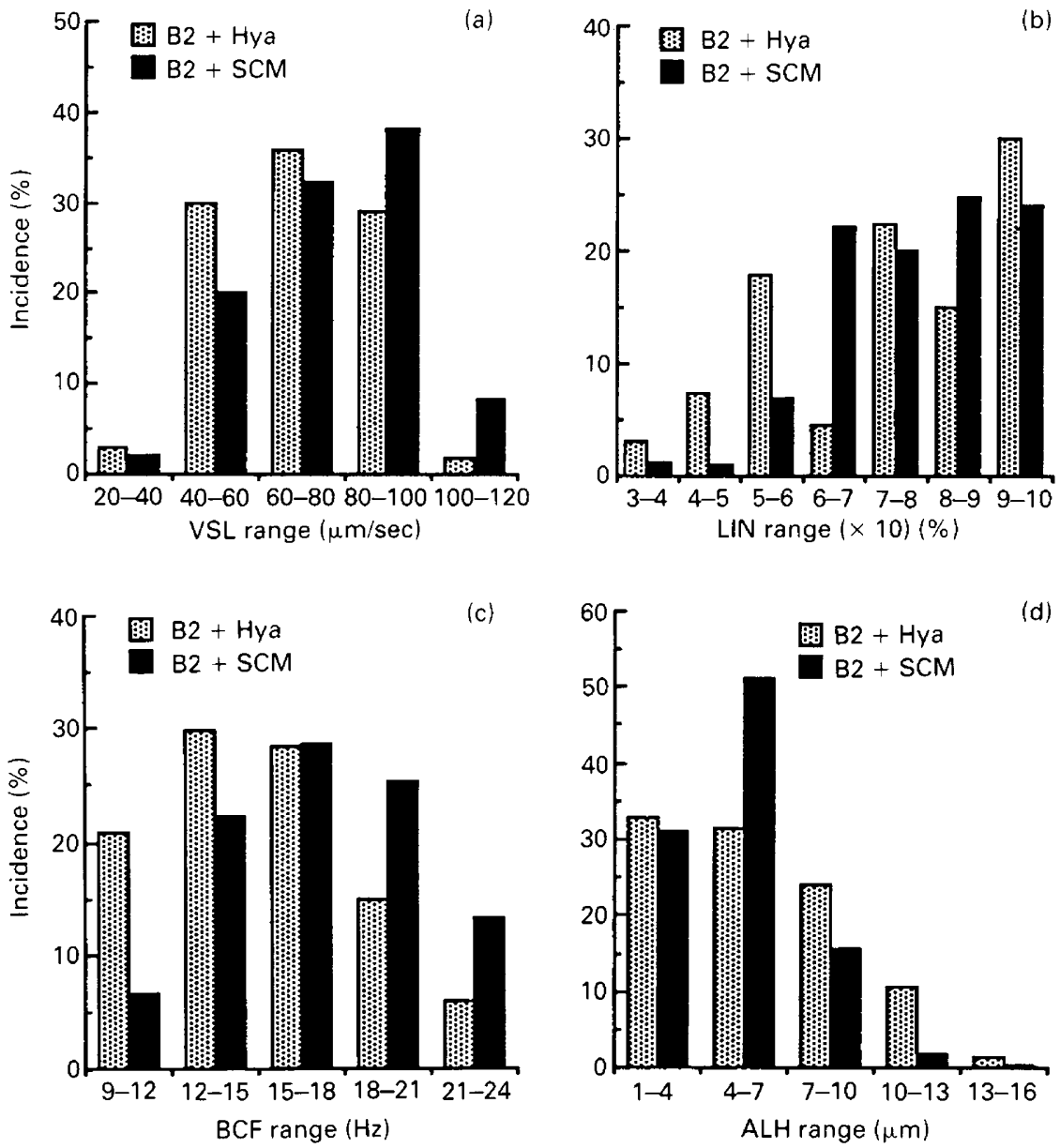

Fig. 2. Bar chart histograms showing frequency distributions of human spermatozoa incubated in B2 medium for $6 \mathrm{~h}$ followed by a 5-min exposure to hyaluronidase preparation alone (B2 + Hya) or to the solubilized cumulus matrix (B2 + SCM) in different ranges of VSL (a), LIN (b), BCF (c) and ALH (d). The ranges are characterized by their lower and upper limits. A motility parameter value is included in the range for which the lower limit $<$ parameter value $\leqslant$ the upper limit.

Effects of h.p.l.c. fractions of the solubilized cumulus intercellular matrix on sperm movement characteristics

When different h.p.l.c. fractions of the solubilized cumulus matrix (Fig. 5) were added to suspensions of spermatozoa preincubated in capacitating medium, the mean values of sperm motility parameters tended to change in the same way as demonstrated with the whole matrix preparations (Table 5). The differences in motility characteristics were significant for fractions 1 to 4 (VSL, BCF and $\mathrm{ALH}$ ) and for fractions 5 and 6 (BCF and $\mathrm{ALH}$ ).

\section{Discussion}

Our data have confirmed the previous findings demonstrating the development of a particular motility pattern, generally referred to as hyperactivated, in a subpopulation of human spermatozoa incubated in vitro under capacitating conditions (Burkman, 1984; Mortimer et al., 1984; Morales 


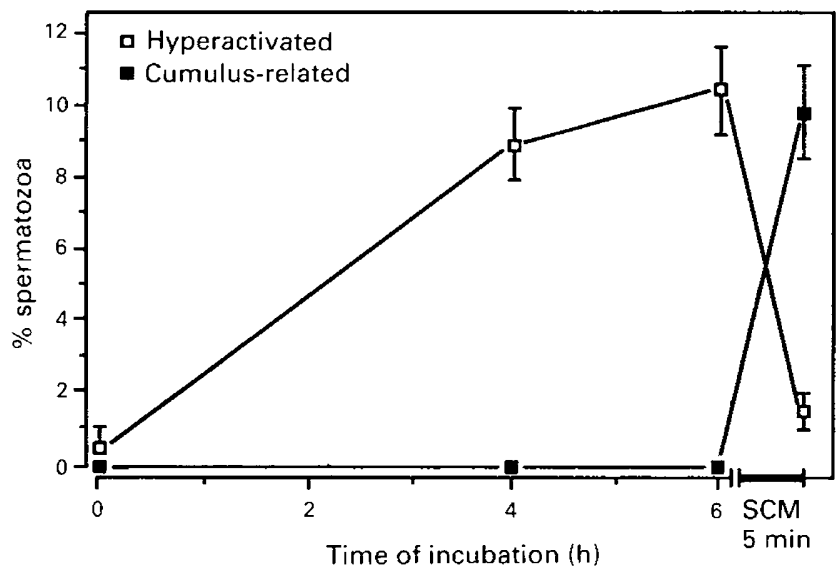

Fig. 3. Changes in the percentage of human spermatozoa showing the hyperactivated and cumulus-related motility patterns during incubation in B2 medium and after subsequent exposure to the solubilized cumulus intercellular matrix (SCM, $5 \mathrm{~min}$ ). Points and vertical bars represent, respectively, means and s.d. for sperm samples from 8 different donors. Criteria of the hyperactivated pattern: VCL $>100 \mu \mathrm{m} / \mathrm{sec}$, VSL $<60 \mu \mathrm{m} / \mathrm{sec}$; BCF $<18 \mathrm{~Hz}$; ALH $>7 \mu \mathrm{m}$. Criteria of the cumulus-related pattern: VCL $>100 \mu \mathrm{m} / \mathrm{sec}$; VSL $>80 \mu \mathrm{m} / \mathrm{sec} ; \mathrm{BCF}>18 \mathrm{~Hz}$; ALH $<5 \mu \mathrm{m}$.



Fig. 4. Typical tracks of human spermatozoa showing non-hyperactivated (a), hyperactivated (b) and cumulus-related (c \& d) movement.

et al., 1988; Robertson et al., 1988), even though there are some differences as to the exact definition of hyperactivation between individual research groups. These differences are mainly related to different measurement conditions which have been shown to influence the perceived characteristics of sperm movement (Mortimer et al., 1988; Mack et al., 1989). By analogy with other mammalian species the hyperactivated sperm subpopulation is believed to represent the capacitated sperm subpopulation. However, the physiological role of this type of sperm motility is still not clear. The 
Table 5. Effect of h.p.l.c. fractions of the solubilized cumulus oophorus intercellular matrix on selected parameters of sperm motility

\begin{tabular}{lccc}
\hline $\begin{array}{l}\text { Sperm } \\
\text { treatment }\end{array}$ & $\begin{array}{c}\text { VSL } \\
(\mu \mathrm{m} / \mathrm{sec})\end{array}$ & $\begin{array}{c}\text { BCF } \\
(\mathrm{Hz})\end{array}$ & $\begin{array}{c}\text { ALH } \\
(\mu \mathrm{m})\end{array}$ \\
\hline Control & $71 \cdot 0 \pm 16 \cdot 9$ & $15 \cdot 2 \pm 2 \cdot 4$ & $6 \cdot 4 \pm 2 \cdot 2$ \\
Fractions 1 \& 2 & $76 \cdot 1 \pm 17 \cdot 1^{\mathrm{a}}$ & $17 \cdot 3 \pm 2 \cdot 4^{\mathrm{b}}$ & $4 \cdot 7 \pm 1 \cdot 8^{\mathrm{c}}$ \\
Fractions 3 \& 4 & $75 \cdot 6 \pm 16 \cdot 7^{\mathrm{a}}$ & $17 \cdot 1 \pm 2 \cdot 5^{\mathrm{b}}$ & $4 \cdot 6 \pm 1 \cdot 9^{\mathrm{c}}$ \\
Fractions 5 \& 6 & $72 \cdot 9 \pm 15 \cdot 9$ & $17 \cdot 4 \pm 2 \cdot 6^{\mathrm{b}}$ & $5 \cdot 8 \pm 2 \cdot 1^{\mathrm{c}}$ \\
Fractions 7 \& 8 & $72 \cdot 2 \pm 17 \cdot 6$ & $15 \cdot 4 \pm 2 \cdot 3$ & $6 \cdot 5 \pm 2 \cdot 3$ \\
Fractions 9 \& 10 & $69 \cdot 8 \pm 16 \cdot 5$ & $15 \cdot 5 \pm 2 \cdot 1$ & $6 \cdot 5 \pm 2 \cdot 2$ \\
\hline
\end{tabular}

Values given are means \pm s.d.

${ }^{\text {a.b,c }}$ Values significantly different from controls: ${ }^{a} P<0.05 ;{ }^{b} P<0.001$;

${ }^{\mathrm{c}} P<0.001$ for Fractions $1 \& 2$ and $3 \& 4 ; P<0.05$ for Fractions 5 \& 6 .

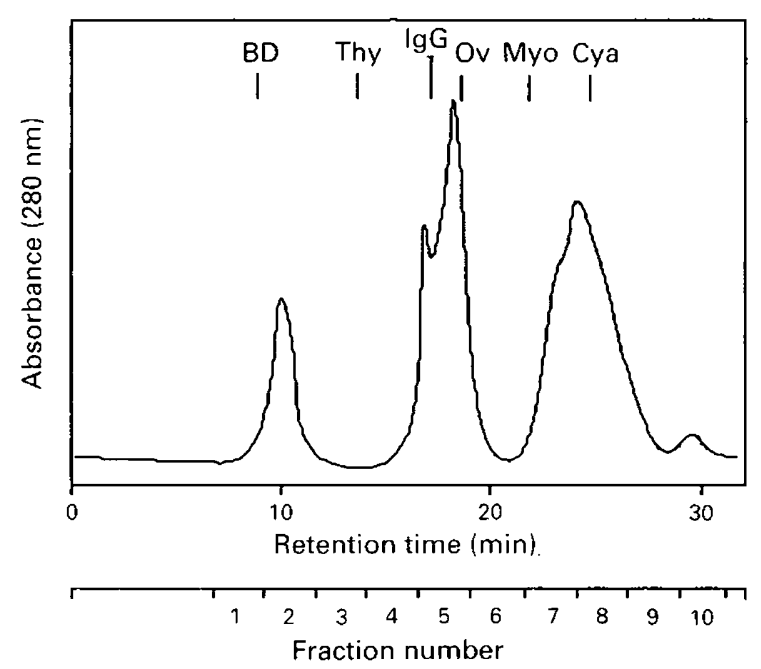

Fig. 5. Chromatogram of solubilized cumulus intercellular matrix. Vertical bars indicate the position of calibration standards: BD, blue dextran; Thy, thyroglobulin; IgG, immunoglobulin G; Ov, ovalbumin; Myo, myoglobin; Cya, cyanocobalamin.

vigorous, whiplash-like movement may be advantageous for liberating capacitated sperm cells from the mucous materials present in various parts of the female reproductive tract. The oviducal isthmus acts as a sperm reservoir in some mammals and it has been demonstrated that hyperactivation of rabbit spermatozoa is associated with their migration from the lower isthmus to the oviducal ampulla (Cooper et al., 1979; Overstreet \& Cooper, 1979). Similar changes can be induced by incubation in capacitating medium in rabbit spermatozoa flushed from the isthmus (Johnson et al., 1981). Cervical crypts have been suggested to represent the main site of sperm storage in the human female reproductive tract (Kremer \& Jager, 1988) and hyperactivated motility of human spermatozoa may thus serve to aid escape from the viscous cervical mucus.

The erratic movement of hyperactivated spermatozoa may also reduce the loss of spermatozoa from the site of fertilization and provide the spermatozoa with a search pattern for the cumulus mass (Suarez et al., 1983). On the other hand, it is more difficult to imagine how this restricted 
progressive motility might help spermatozoa to enter the cumulus oophorus and move within it in a centripetal fashion towards the oocyte. The present results have shown that most of the spermatozoa within the cumulus mass display a very linear motility, different from the hyperactivated pattern. The persistence of great amplitudes of lateral head displacement in some spermatozoa within the cumulus matrix is associated with a rather ineffective movement and a marked drop of flagellar beat frequency. This may be due to great loss of energy consumed by such spermatozoa during forced vast lateral excursions of the sperm head in the extremely viscous environment of the cumulus oophorus. Alternatively, it cannot be excluded that these spermatozoa are actually responding to less penetrable regions of the cumulus matrix. Local heterogeneity in cumulus mechanical resistance is probably a factor affecting the kinematics of sperm movement in the hamster cumulus matrix (Drobnis et al., 1988).

The motility pattern of human spermatozoa within the cumuli oophori observed in this study is different from that described for rabbit (Suarez et al., 1983) and rat (Shalgi \& Phillips, 1988) spermatozoa that have entered the cumulus oophorus in vivo and which retain the hyperactivated motility. This difference must be interpreted with caution since these in-vivo studies involved quite different physiological conditions. In particular, we have analysed the motility of spermatozoa after a very short duration of contact with the cumulus. Therefore the motility pattern observed may represent an early response of spermatozoa to the exposure to cumulus factors which accelerates their passage through this egg coat. If spermatozoa remain in the cumulus for longer periods, they may return to the hyperactivated motility pattern. A biphasic pattern characterized by oscillations between whiplash and progressive motility is typical of the hyperactivated state in rabbit spermatozoa and the durations for either phase may be as long as several minutes (Johnson et al., 1981). Unlike the above two species, however, the movement of hamster spermatozoa becomes more linear within the cumulus (Katz et al., 1986; Corselli \& Talbot, 1987; Drobnis et al., 1988) in a way similar to that described in this study. The possibility of interspecies differences in sperm reactivity with components of the cumulus oophorus cannot be excluded.

Our experiments using the solubilized cumulus intercellular matrix have suggested that there may be a direct effect of some components of the cumulus intercellular material on sperm motility, apart from the purely mechanical one given by the physical properties of this milieu. In fact, a new sperm subpopulation, characterized at the same time by very high curvilinear velocity and low amplitude of lateral head displacement, resulting in an extraordinarily high progressive velocity, appears during short incubation with the solubilized cumulus matrix. A considerable increase in the mean value of beat cross frequency is another typical sign of this subpopulation. This linear and extremely progressive movement, never observed in fresh spermatozoa or in those incubated in capacitating medium, may facilitate sperm penetration through the cumulus oophorus. On the other hand, the major differences between the intact cumulus oophorus and the solubilized cumulus matrix preparation, involving viscosity, presence or absence of cumulus cells and the relative concentrations and size of polysaccharides, make it extremely difficult to compare the sperm movements generated in each preparation. The principal problem to be solved is the relationship between the subpopulation of spermatozoa displaying the cumulus-related motility pattern in the low-viscosity medium and those showing a similar character of movement within the intact cumulus matrix.

The appearance of this specific motility pattern of spermatozoa preincubated in capacitating medium and then exposed to the solubilized cumulus matrix is accompanied by a marked reduction of the subpopulation of spermatozoa showing the hyperactivated type of motility. This relationship suggests that the spermatozoa with extremely swift and linear movement appearing in the presence of the solubilized cumulus matrix are recruited from the hyperactivated sperm subpopulation. This hypothesis is supported by the absence of response to the solubilized cumulus matrix, in terms of the development of this particular type of movement, in short-term incubation, non-capacitated sperm populations. It has been shown in various mammalian species that non-capacitated spermatozoa lack the ability to enter the cumulus (for review see Yanagimachi, 1988). However, other 
factors than the character of sperm movement may be responsible for this inability, particularly those concerning the readiness of spermatozoa to undergo the acrosome reaction which must occur in synchrony with sperm passage through the egg vestments to ensure normal penetration (Tesaŕik et al., 1988; Tesařík, 1989).

The fact that several different h.p.l.c. fractions of the solubilized cumulus intercellular matrix are capable of inducing the changes in sperm motility characteristics typical of the cumulus-related motility may be explained in several ways. This effect may be produced by different types of molecules acting synergistically in the intact cumulus but capable of exerting the same effect, under the experimental conditions used in this study, independently of each other. Alternatively, the same active substance may be part of a large macromolecule and remain functional after its partial enzymic degradation by the action of enzymes originating from cumulus cells, from spermatozoa, or from the hyaluronidase preparation used for cumulus dispersion. Further studies are needed to identify and characterize the cumulus components involved in modifying human sperm motility.

In conclusion, this study has demonstrated that human spermatozoa reacting with a human cumulus oophorus in vitro assume a specific type of motility. This specific motility, denoted as cumulus-related, is characterized by a very rapid, linear and progressive movement with high frequency of flagellar beat and low lateral excursions of the sperm head. The cumulus-related motility is induced by factors present in the cumulus intercellular matrix which probably act on those spermatozoa that have previously achieved the hyperactivated motility pattern. It would be of interest to determine whether defects of this mechanism, caused either by deficiency of cumulus oophorus preovulatory development or by abnormal sperm responsiveness, can be implicated in some cases of human infertility.

We thank IMV (L'Aigle, 61300 France) for the kind loan of the HTM (Hamilton Thorn, Inc., Danvers, MA, USA) motility analyser.

\section{References}

Burkman, L.J (1984) Characterization of hyperactivated motility by human spermatozoa during capacitation: comparison of fertile and oligozoospermic sperm populations. Archs Androl. 13, 153-165.

Cooper, G.W., Overstreet, J.W. \& Katz, D.F. (1979) The motility of rabbit spermatozoa recovered from the female reproductive tract. Gamete Res. 2, 35-42.

Corselli, J. \& Talbot, P. (1987) In vitro penetration of hamster oocyte-cumulus complexes using physiological numbers of spermatozoa. Devl. Biol. 122, $227-242$.

Drobnis, E.Z., Yudin, A.I., Cherr, G.N. \& Katz, D.F. (1988) Kinematics of hamster sperm during penetration of the cumulus cell matrix. Gamete Res. 21, 367-383.

Johnson, L.L., Katz, D.F. \& Overstreet, J.W. (1981) The movement characteristics of rabbit spermatozoa before and after activation. Gamete Res. 4, 275-282.

Katz, D.F., Cherr, G.N. \& Lambert, H. (1986)The evolution of hamster sperm motility during capacitation and interaction with the ovum vestments in vitro. Gamete Res. 14, 333-346.

Kremer, J. \& Jager, S. (1988) Sperm-cervical mucus interaction, in particular in the presence of antispermatozoal antibodies. Hum. Reprod. 3, 69-73.

Laufer, N., Tarlatzis, B.C., DeCherney, A.H., Masters, J.T., Haseltine, F.P., MacLusky, N. \& Naftolin, F. (1984) Asynchrony between human cumulus-corona cell complex and oocyte maturation after human menopausal gonadotropin treatment for in vitro fertilization. Fert. Steril. 42, 366-372.

Mack, S.O., Tash, J.S. \& Wolf, D.P. (1989) Effect of measurement conditions on quantification of hyperactivated human sperm subpopulations by digital image analysis. Biol. Reprod. 40, 1162-1169.

Marrs, R.P., Saito, H., Yee, B., Sato, F. \& Brown, J. (1984) Effect of variation of in vitro culture techniques upon oocyte fertilization and embryo development in human in vitro fertilization procedures. Fert. Steril. 41, 519-523.

Morales, P., Overstreet, J.W. \& Katz, D.F. (1988) Changes in human sperm motion during capacitation in vitro. J. Reprod. Fert. 83, 119-128.

Mortimer, D., Courtot, A.M., Giovangrandi, Y., Jeulin, C. \& David, G. (1984) Human sperm motility after migration into, and incubation in, synthetic media. Gamete Res. 9, 131-144.

Mortimer, S., Serres, C., Mortimer, S.T. \& Jouannet, P. (1988) Influence of image sampling frequency on the perceived movement characteristics of progressively motile human spermatozoa. Gamete Res. 20, 313-327.

Overstreet, J.W. \& Cooper, G.W. (1979) Effect of ovulation and sperm motility on the migration of rabbit spermatozoa to the site of fertilization. J. Reprod. Fert. 55, 53-59.

Robertson, L., Wolf, D.P. \& Tash, J.S. (1988) Temporal changes in motility parameters related to acrosomal 
status: identification and characterization of populations of hyperactivated human sperm. Biol. Reprod. 39, 797-805.

Shalgi, R. \& Phillips, D.M. (1988) Motility of rat spermatozoa at the site of fertilization. Biol. Reprod. 39, 1207-1213.

Suarez, S.S., Katz, D.F. \& Overstreet, J.W. (1983) Movement characteristics and acrosomal status of rabbit spermatozoa recovered at the site and time of fertilization. Biol. Reprod. 29, 1277-1287.

Tesařik, J. (1989) Appropriate timing of the acrosome reaction is a major requirement for the fertilizing spermatozoon. Hum. Reprod. 4. (In press).

Tesařík, J., Drahorád, J. \& Peknicova, J. (1988) Subcellular immunochemical localization of acrosin in human spermatozoa during the acrosome reaction and zona pellucida penetration. Fert. Steril. 50, 133-141.
Testart, J., Lassalle, B. \& Frydman, R. (1982) Apparatus for the in vitro fertilization and culture of human oocytes. Fert. Steril. 38, 372-375.

Testart, J., Belaisch-Allart, J.C., Forman, R., Gazengel, A., Strubb, N., Hazout, A. \& Frydman, R. (1989) Influence of different stimulation treatments on oocyte characteristics and in vitro fertilizing ability. Hum. Reprod. 4, 192-197.

World Health Organization (1987) Laboratory Manual for the Examination of Human Semen and Semen Cervical Mucus Interaction, 2nd edn. Cambridge University Press, Cambridge.

Yanagimachi, R. (1988) Mammalian fertilization. In The Physiology of Reproduction, pp. 135-185. Eds E. Knobil \& J. Neill. Raven Press, New York.

Received 25 August 1989 